\title{
Preface: evidence-based clinical practice guidelines for CKD—an abridged English version
}

\author{
Kenjiro Kimura
}

Published online: 10 May 2014

(C) Japanese Society of Nephrology 2014

\section{Origins of the guidelines}

The concept of chronic kidney disease (CKD), first proposed in 2002 in the United States, has now become accepted around the world. CKD is a risk factor not only for progression to end-stage kidney disease but also for the onset or progression of cardiovascular diseases. As a result, early detection and treatment of CKD are now being prioritized as urgent concerns. The Japanese Society of Nephrology (JSN) has long been focused on CKD, and in September 2007, we published the "Clinical Practice Guidebook for the Diagnosis and Treatment of CKD" (Guidebook for CKD) (Chairperson: Yasuhiko Iino) for non-specialists. Subsequently, in March 2009, the JSN published the "Evidence-Based Clinical Practice Guidelines for CKD 2009" (Guidelines for CKD 2009) (Chairperson: Sei Sasaki) for kidney specialists. The difference between these two publications is that the Guidebook for CKD was based on a general consensus of the committee, whereas the Guidelines for CKD 2009 was based on the rigorous evaluation of clinical evidence.

The Kidney Disease Improving Global Outcomes (KDIGO) later published a revised version of the severity classification system (heat map) for CKD based on a combination of the glomerular filtration rate (GFR) and urinary protein (albumin) values. In response to that revision, the JSN prepared the Guidebook for CKD 2012 (Chairperson: Enyu Imai), which adopted a new CKD classification system. This new CKD classification system in Japan took into consideration that the Japanese health

K. Kimura $(\bowtie)$

Chairperson, JSN Committee for Evidence-Based Clinical

Practice Guidelines for CKD, Tokyo, Japan

e-mail: 10471122kimura@gmail.com insurance system recognizes quantitative measurement of urinary albumin only for diabetic nephropathy, and thus urinary protein was included in the urinary albumin category. Subsequently, the JSN decided to revise the Guidelines for CKD in light of both the revised Guidebook for CKD and the clinical evidence that has accumulated since the publication of the Guidelines for CKD 2009. In order to implement that decision, the JSN established the Committee for the Revision of the Guidelines for CKD within its Academic Committee.

\section{The intended purpose, anticipated users, and predicted social significance of the guidelines}

The Guidelines for CKD responds to clinical questions (CQs) that arise when kidney specialists are caring for CKD patients. In cases where the response includes a treatment option, a recommendation grade for that treatment has been assigned based on the level of evidence supporting the use of the treatment. Therefore, the combined use of both the Guidelines for CKD 2013 and the Guidebook for CKD 2012 will provide non-specialists and even non-physician health care providers with a deeper understanding of CKD clinical practice.

Evidence obtained from the published literature provides information, but it does not replace the individual physician's expertise and experience. It is the physician, as a professional, who must decide whether the statements in the Guidelines apply to individual patients, and how exactly to use that information. The needs of the times have shifted from one-size-fits-all to tailor-made medical approaches. Thus, clinical practice guidelines must not force doctors to take a cookie-cutter approach. Accordingly, these Guidelines were not prepared with the 
intention of directly dictating how physicians should approach the treatment of CKD patients, but rather with the hope that they will serve as a resource for physicians, assisting them to make discretionary judgments on the optimum treatment for their individual patients. It should also be clearly stated that these Guidelines do not claim to set forth criteria as a basis for decisions taken during medical disputes or litigations.

\section{Patients within the scope of the guidelines}

The Guidelines are intended as a reference during the treatment of non-dialysis CKD patients in all age strata (G1-G5 categories), regardless of gender or the presence or absence of complications. Children are addressed in Chapters 16 (diagnosis) and 17 (treatment), and elderly patients are addressed separately in Chapter 20. Renal replacement therapy is covered in Chapters 18 (dialysis) and 19 (renal transplantation), but the discussion is centered on problems encountered when non-dialysis CKD patients are switched to renal replacement therapy. These Guidelines are focused on non-dialysis CKD patients and exclude, in principle, dialysis and renal transplant patients.

\section{Evidence levels and recommendation grades}

Evidence was classified into six levels based on the study design, and was arranged roughly from the most reliable study type (Level 1) to the least reliable (Level 6). These levels do not necessarily represent rigorous scientific standards; they are intended for use as a convenient reference for quickly assessing the significance of various clinical data during the physician's decision-making process.

Evidence levels

Level 1: Systematic review/meta-analysis.

Level 2: At least one randomized controlled trial (RCT).

Level 3: A non-randomized controlled trial.

Level 4: An analytical epidemiologic study (cohort study or case-control study) or a single-arm intervention study (no controls).

Level 5: A descriptive study (case report or case series).

Level 6: Opinion of an expert committee or an individual expert, which is not based on patient data.

However, for a systematic review/meta-analysis, the evidence level was decided based on the designs of the underlying studies. If the underlying study designs were mixed, the lowest level underlying study was used to determine the overall evidence level. For example, a metaanalysis of cohort studies would be Level 4 , but the same Level 4 would also be assigned to a meta-analysis including both RCTs and cohort studies.

In addition, a decision based on committee consensus was that all sub-analyses and post hoc analyses of RCTs should be categorized at evidence Level 4. Accordingly, it was decided that the evidence level of findings representing the primary endpoints of an RCT would be Level 2, but the evidence level of findings determined via a sub-analysis or post hoc analysis of that RCT would be Level 4 .

When a statement related to a certain treatment was presented, consideration was given to the level of the evidence serving as the basis of that statement, and a recommendation grade was assigned as outlined below:

Recommendation grades

Grade A: Strongly recommended because the scientific basis is strong.

Grade B: Recommended because there is some scientific basis.

Grade C1: Recommended despite having only a weak scientific basis.

Grade C2: Not recommended because there is only a weak scientific basis.

Grade D: Not recommended because scientific evidence shows the treatment to be ineffective or harmful.

If we found only a weak scientific basis for a certain statement concerning a treatment, the members of the committee discussed the matter and decided on $\mathrm{C} 1$ or $\mathrm{C} 2$ for the recommendation grade. Thus, discrimination between $\mathrm{C} 1$ and $\mathrm{C} 2$ statements was based on expert consensus.

\section{Publication and future revisions}

The Guidelines were published in the Japanese-language journal of the JSN and concurrently released as a Japaneselanguage book (by Tokyo Igakusha, Tokyo). The Guidelines were also uploaded to the homepage of the JSN.

At present, CKD-related evidence is being rapidly accumulated, and this new evidence will necessitate the preparation of an updated version of the Guidelines in 3-5 years. A certain degree of turnover in the membership of the revision committee will be required in order to ensure the impartiality of the Guidelines. 